\title{
Research on Higher Education of Traditional Chinese Medicine in Southeast Asia in the Post-epidemic Era
}

\author{
Jing $\mathrm{Li}^{1}$, Xiaoxing Sun ${ }^{1}$ and $\mathrm{Li}$ Zhou ${ }^{1, *}$ \\ ${ }^{1}$ School of foreign languages, Hubei University of Chinese Medicine, Wuhan, Hubei 430065, China \\ *Corresponding author. Email: kerryqq@126.com
}

\begin{abstract}
This article intends to research the current development and challenges in higher education of Traditional Chinese Medicine (TCM) in China and Southeast Asia during the pandemic, and puts forward corresponding measures. Suggestions are: expanding enrollment, summarizing online teaching experience, inventing the "Internet plus TCM" education model. Through these measures, deeper thoughts and better methods should be explored. This article aims to ensure the quality of teaching and satisfy the needs of the development of TCM education, and to promote the development of TCM in Southeast Asia and the implementation of the "going out " strategy of TCM.
\end{abstract}

Keywords: Southeast Asia; Traditional Chinese medicine; Higher education; Post-epidemic era

\section{INTRODUCTION}

Southeast Asia is a region located between Asia and Oceania, the Pacific and Indian oceans, it consists of 11 countries and adjoins China. Back in the Qin and Han Dynasties, the turbulent situation caused by continuous wars in the Central Plains region forced people to move southward. The culture of the Central Plains was then introduced to Southeast Asian countries, among which the culture of Traditional Chinese medicine was included. With adjacent geographical location and political support, Southeast Asia and China shared a long history of exchanges in Traditional Chinese Medicine. After the "Belt and Road" initiative and the establishment of the China-ASEAN community of shared destiny, TCM has gained new development opportunities in Southeast Asia, with China as the center and Japan, South Korea and Southeast Asian countries as the periphery, attempting to achieve extensive exchanges and cooperation of TCM culture.

\section{CURRENT DEVELOPMENT IN HIGHER EDUCATION OF TCM IN CHINA AND SOUTHEAST ASIA}

By the end of 2017, China had 43 universities of TCM, along with 110 universities of western medicine and 164 more universities with TCM majors. [1] In recent years, the number of students from Southeast Asian countries who study TCM in China has been increasing due to geographical advantages, sound economic development, shared cultural foundation, large population base and so forth. Since 1957, the universities of TCM in China began to enroll oversea students to study TCM [2]. According to the statistics, compared with Africa, Europe, North America, South America and Australia, the number of Asian students studying Chinese medicine in China ranks first. In the past decade, the number of overseas students in Chinese medicine colleges and universities has maintained a steady growth. The number of overseas students studying TCM in China reached 8,777 in 2019, and among them 6,785 students were from Asia (See fig.1).

(Data from China Statistical Yearbook of Chinese Medicine (2010-2019)).

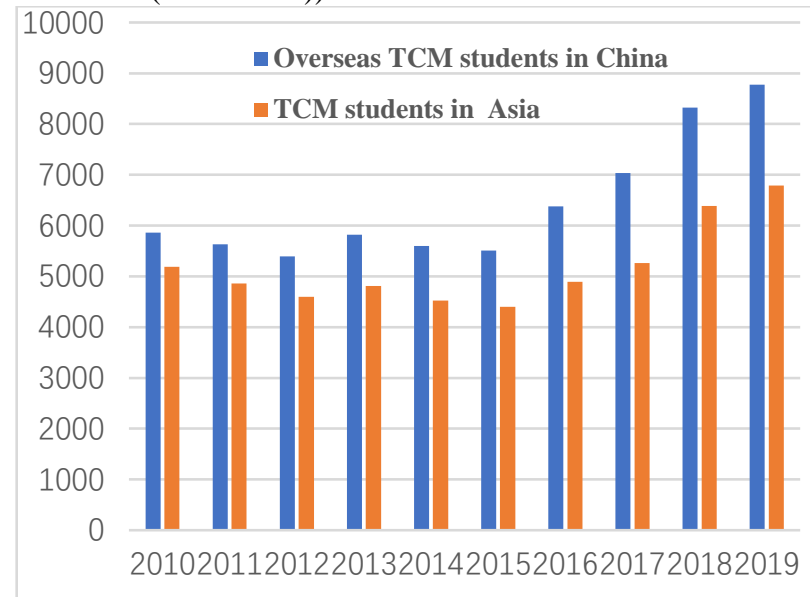

Figure 1. Basic information on Overseas Students in universities of TCM in China from 2010 to 2019

Generally speaking, there are two ways for TCM to go abroad: Developing international higher education of TCM in China and exploring the potential of TCM higher education overseas. According to the current economic and educational development of Southeast Asian countries, development of TCM higher education can be generally categorized into three groups. Singapore, Thailand and Malaysia rank first, showing a leading role in TCM higher 
education and owning larger scale of students. TCM curriculum system and talent training mode in Singapore and Thailand are more comprehensive as they started TCM courses earlier than other countries in that region. The Malaysian government approved the establishment of TCM higher education in 2009, and since then the cooperation with TCM colleges in China were held up frequently, which has promoted the rapid development of TCM education in Malaysia. The second level of international TCM higher education is still in stage of development, including countries like Vietnam and Indonesia. At present, six medical schools in Vietnam have set up TCM course. Moreover, with the frequent exchanges between China and Vietnam in recent years, the Ministry of Health in Vietnam would send Vietnamese doctors to China to learn traditional Chinese medicine Every year [3]. The relationship between China and Indonesia improved after the reform and opening up, since then TCM has gained the legal status in Indonesia. Statistics showed that the number of TCM acupuncturists in Indonesia has growing increase in recent years [4]. The third level of international TCM higher education is relatively underdeveloped, of which the representative countries are Philippines, Myanmar, Laos, Cambodia, Brunei and Timor-Leste. Currently, there are no TCM colleges and universities in third-level countries, and their study channel of TCM is limited to TCM educational and academic activities, their development of TCM education is temporarily in a stagnating stage [5].

To sum up, certain imbalance of TCM higher education in various countries is existing due to the political, economic and cultural differences in Southeast Asia. In countries with rapid economic development and sound political conditions, such as Malaysia, Thailand and Vietnam, TCM higher education is relatively more complete and TCM colleges and universities have relatively larger scale. On the contrary, the development of TCM higher education in other Southeast Asian countries lags behind and the scale of schooling is relatively small. On the whole, the talent training mode of TCM in Southeast Asia is not complete and the teaching system is not perfect, and the outbreak of COVID-19 has exposed the shortcomings and deficiencies of TCM higher education system in Southeast Asia.

\section{CHALLENGES OF TCM HIGHER EDUCATION IN SOUTHEAST ASIA DURING THE EPIDEMIC}

\subsection{Challenges faced by international higher education of TCM in China}

\subsubsection{The decreased number of international students}

The National Program for Medium - and Long-term Education Reform and Development 2010-2020 proposed the goal of implementing "Study in China" program to further expand the scale of foreign students, however, due to the outbreak of COVID-19, the international exchanges were forced to be weaken, which has made the goal difficult to be achieved. Moreover, as the epidemic firstly broke out in China, some foreign media released false statements about China, which has caused lots of international students evacuated from China. For overseas students, as well as students who plan to study in China in the future, their willingness to China are temporarily decreased. Therefore, the number of international students in China decreased significantly.

\subsubsection{Scarcity of online teaching experience}

Since the outbreak of COVID-19 pandemic, online education has become the common teaching mode around the world, with teachers and students finishing their teaching tasks through online platforms. But under the pressure from the first large-scale online education, schools in China and around the world, including in Southeast Asia, are paying efforts to seek a way adapting the mode. On one hand, the disadvantages of online teaching such as network instability and poor interaction between teachers and students affected the effect of both teaching and learning. On the other hand, for senior teachers who are not familiar with online teaching platform operation, it takes time for them to adapt to the online teaching mode. Moreover, the teaching content and teaching methods need to be adjusted with the change of the teaching mode. The teachers need to better complete the teaching objectives and let students better participate in the classroom. These existing problems made it hard to guarantee the study quality and efficiency of international TCM students during the epidemic.

\subsection{Challenges of TCM higher education in Southeast Asia}

\subsubsection{The imbalance of Internet Penetration}

The internet penetration rate in Southeast Asian countries is uneven. Statistics show that Singapore, Vietnam and Thailand have faster Internet development and higher penetration rate. By 2009, Singapore had $99 \%$ broadband Internet coverage. Vietnam currently has 22.779 million Internet users, and Internet has become its main channel for obtaining information. Thailand has nearly 18 million Internet users by 2010. The network construction in Laos started fairly late, but it has great potential for development [6]. Other Southeast Asian countries have low Internet penetration rate and high Internet cost. For example, Myanmar and Timor-Leste are still at a low level of Internet development. During the epidemic, it is difficult for countries with low Internet penetration to conduct online education in an orderly manner, which is not 
conducive to the development of TCM higher education in Southeast Asia.

\subsubsection{Difficulty in clinical practice instruction}

Different from other majors, TCM is a practical course. TCM lessons generally combine theoretical knowledge with practical operation, while online teaching during the epidemic mostly focuses on theoretical knowledge. Moreover, if the teachers only use video, audio and live broadcast to teach clinical practice skills, such as acupuncture, massage and other clinical practice skills, the teaching effect will be greatly reduced. Thus, improving students' clinical practice skill is another unsettled problem.

\section{TRANSITIONAL STRATEGIES OF TCM HIGHER EDUCATION IN SOUTHEAST ASIA IN THE POST-EPIDEMIC ERA}

\subsection{Transitional strategies of TCM international Higher education in China}

\subsubsection{Expand enrollment of overseas students}

Southeast Asian countries are geographically and culturally close to China, which indicates that Southeast Asian students are convenient to study TCM in China and has a positive sense of identity to TCM. Based on these favorable factors, China should take itself as the center and Southeast Asian countries as the periphery to expand the training scale of TCM talents. According to data from the Ministry of Education in 2017, in the medical education system, the number of students majoring in medicine was 1.244 million, while the number of students majoring in Traditional Chinese medicine was 170,000, accounting for only 13.7 percent of the medical education scale [7]. With the sudden outbreak of the epidemic, medical talents, especially TCM talents, are in a shortage, which urgently requires China to expand the scale of TCM talent training. To make this happen, Firstly, based on the geographical and cultural advantages of Southeast Asia, we should increase the enrollment of overseas students majoring in TCM, and promote TCM dissemination overseas. Second, implement the "equal emphasis on traditional Chinese medicine and western medicine" strategy. Add more TCM courses in domestic colleges and universities of western medicine, incorporate the TCM course into the teaching system of western medicine and expand the scale of TCM learners. Third, cultivate and develop compound TCM talents. China should cultivate and explore the teaching mode of "TCM + English" as students from Southeast Asian countries have good command of English. Also, train more TCM professionals who are proficient in
English, and enhance the influence of TCM in Southeast Asia.

\subsubsection{Promotion of online teaching practice}

Epidemic prevention and control cannot be ignored even in the post-epidemic era, it can be foreseen that online teaching will become a new normal in the future. During the process, teachers are facing the role transition. Thus, they should equip the ability of summarizing the online teaching experience and adapt to the online teaching mode as soon as possible. Students should also quickly adapt to online teaching mode, giving full play to their own positive initiative and self-control, and making full use of online learning resources to maximize the advantages of online learning. The teaching concept of "students first, teachers second" should also be thoroughly implemented in online teaching during the epidemic period.

\subsection{Transitional strategies of TCM higher education in Southeast Asia}

\subsubsection{Promoting the Internet penetration rate, exploring the "Internet + TCM" education model}

China has provided various assistance to countries along the Belt and Road since the Belt and Road Initiative was put forward in 2013. The assistance was not limited to financial assistance, but also technical support as well. Through the Belt and Road Initiative, China has made great contributions to the Internet development in Southeast Asia. In recent years, Southeast Asia has increasing Internet penetration rate, especially in second and third level countries. With the implementation of the Belt and Road Initiative and the popularization and application of the Internet, TCM will be introduced to more countries and regions. The COVID-19 outbreak highlights the importance of "Internet +". At present, the epidemic is far from over, epidemic prevention and control will become normalized, and online teaching will become a common teaching method. The development and utilization of MOOCs is one of the effective teaching platform during the epidemic. China should develop English MOOCs for TCM majors and build a teaching platform for TCM dissemination through the Internet. Through the MOOC and live online teaching platform, high-quality resources can be shared, and TCM education can be promoted in Southeast Asian countries in an efficient and convenient way.

\subsubsection{Developing "online + offline" clinical practice teaching mode}

In the 20th century, a new, practical and interactive virtual reality technology has been developing and widely used in 
various fields. It combines computer and artificial intelligence technologies to form three-dimensional dynamic vision for simulation, and thus achieved immersive and interactive experience. The clinical practice of TCM can use virtual reality to carry out online clinical teaching, and students can use virtual reality technology to observe and practice in a close distance in virtual scenes. During the epidemic, the use of emerging technologies is conducive to the orderly teaching of traditional Chinese medicine, but it still has its limitation, so offline clinical practice is also of particularly important. In the post-epidemic era, people can carry out normal medical activities when protective measures are taken. Therefore, Southeast Asia countries should make use of existing local resources, and provide a platform for students to practice, train and experiment in the community. By applying the theoretical knowledge learned online, students can not only improve their practical operation ability, but also play a role of spreading knowledge and culture of traditional Chinese medicine in the community, which is conducive to the spread and development of traditional Chinese medicine culture in Southeast Asia.

\section{CONCLUSION}

In the process of fighting against the epidemic, TCM has played a huge role in the prevention and treatment of the epidemic, which gives TCM researchers and learners opportunities and confidence. As the old saying goes "Wine is not afraid of deep alley", we should take ourselves as the center, enhance our confidence in TCM, develop the higher education and internationalization of TCM, and take Southeast Asia as peripheral to expand the number of TCM talents, explore appropriate mode to better train the Southeast Asian TCM talents, promote its transition and upgrade. In order to assist TCM to go further abroad and bring benefits to the world.

\section{ACKNOWLEDGMENT}

This work was supported by the educational and research project of Hubei University of Chinese Medicine in 2020Research on Higher Education of Traditional Chinese Medicine in Southeast Asia in the Post-epidemic Era.

\section{REFERENCES}

[1] Jianfei Zhu, "Traditional education helps build a community with a shared future for mankind," China net of Traditional Chinese Medicine.

http://www.cntcm.com.cn/news.html?aid=146554 (In Chinese)

[2] Fuchun Si, Xuejie Song, "The current situation and thinking about the education of foreign students in Traditional Chinese Medicine colleges and universities", TCM Research, vol. 25, pp.5-7, June 2012. DOI: 10.3969/j.issn.1001-6910.2012.06.003 (In Chinese)

[3] Weimin Zhou, Dongpei Hu, "The influence of Traditional Chinese Medicine on Vietnamese traditional medicine--Also on the current status of Vietnamese traditional medicine", Chinese Medicine and Culture, vol. 2, pp.53-56, August 2013. DOI: 10.16307/j.1673-6281.2013.02.012 (In Chinese)

[4] Rui Wang, Junlong Shen, "A brief analysis on the spread and development of Traditional Chinese Medicine in Southeast Asia", World Chinese Medicine, vol. 10, pp.1976-1979, December 2015. DOI: 10.3969 /j.issn.1673-7202.2015.12.039 (In Chinese)

[5] Weiwei Chong, Xiang Wen, "Summary of Chinese medicine education in Southeast Asia from the perspective if internationalization", Asia-Pacific Traditional Medicine, vol. 13, pp.1-3, November 2017. DOI: 10. 11954/ytctyy. 201721001 (In Chinese)

[6] Lina Yang, Li Shang, "An approach to realize "Internet + TCM" in Southeast Asia under the Belt and Road Initiative", Lishizhen Medicine and Materia Medica Research, vol. 31, pp.1014-1015, 2020. DOI: 10.3969/j.issn.1008-0805.2020.04.077 (In Chinese)

[7] Reflection on higher education of Traditional Chinese Medicine under the background of epidemic situation, http://www.satcm.gov.cn/hudongjiaoliu/guanfangweixin /2020-04-07/14507.html (In Chinese) 